\title{
Lactic acid bacteria that activate immune gene expression in Caenorhabditis elegans can antagonise Campylobacter jejuni infection in nematodes, chickens and mice
}

Jin Xing ${ }^{1,2}$, He Yufeng ${ }^{1,2}$, Zhou Yonghua ${ }^{3}$, Chen Xiaohua ${ }^{4}$, Lee Yuan-kun ${ }^{5}$, Zhao Jianxin 1,2,6,7, Zhang Hao 1,2,7,8,9, Chen Wei ${ }^{1,2,8,10}$ and Wang Gang ${ }^{1,2,6,7^{*}}$ (D)

\begin{abstract}
Background: Campylobacter jejuni is the major micro-bacillary pathogen responsible for human coloenteritis. Lactic acid bacteria (LAB) have been shown to protect against Campylobacter infection. However, LAB with a good ability to inhibit the growth of $C$. jejuni in vitro are less effective in animals and animal models, and have the disadvantages of high cost, a long cycle, cumbersome operation and insignificant immune response indicators. Caenorhabditis elegans is increasingly used to screen probiotics for their anti-pathogenic properties. However, no research on the use of $C$. elegans to screen for probiotic candidates antagonistic to $C$. jejuni has been conducted to date.

Results: This study established a lifespan model of $C$. elegans, enabling the preselection of $L A B$ to counter $C$. jejuni infection. A potential protective mechanism of $L A B$ was identified. Some distinct $L A B$ species offered a high level of protection to $C$. elegans against $C$. jejuni. The $L A B$ strains with a high protection rate reduced the load of $C$. jejuni in C. elegans. The transcription of antibacterial peptide genes, MAPK and Daf-16 signalling pathway-related genes was elevated using the $L A B$ isolates with a high protection rate. The reliability of the lifespan model of $C$. elegans was verified using mice and chickens infected with $C$. jejuni.
\end{abstract}

Conclusions: The results showed that different $\angle A B$ had different abilities to protect $C$. elegans against $C$. jejuni. $C$. elegans provides a reliable model for researchers to screen for $L A B$ that are antagonistic to $C$. jejuni on a large scale.

Keywords: Lactic acid bacteria, Campylobacter jejuni, Caenorhabditis elegans, Life-span, Immune gene

\section{Background}

Infection by pathogenic Campylobacter may result in symptoms such as bloody diarrhoea, abdominal pain and fever. In many countries, Campylobacter species is the major microbacillary pathogen responsible for human

\footnotetext{
* Correspondence: wanggang@jiangnan.edu.cn

'State Key Laboratory of Food Science and Technology, Jiangnan University, Wuxi 214122, P. R. China

${ }^{2}$ School of Food Science and Technology, Jiangnan University, Wuxi 214122, P. R. China

Full list of author information is available at the end of the article
}

coloenteritis [1]. In under developed countries, diarrhoeic disease is 10 times more likely to result from Campylobacter infection than from infection with Escherichia coli O157: H, Shigella species or Salmonella species [2, 3]. Some peripheral neuropathies, such as Guillain-Barré syndrome and Miller Fieher syndrome, are long-term consequences of Campylobacter infection [2]. Campylobacter jejuni (C. jejuni) is also one of the primary reasons for microbacillary food-borne disease in some developed countries [3]. To date, all therapies for

C C The Author(s). 2021 Open Access This article is licensed under a Creative Commons Attribution 4.0 International License, which permits use, sharing, adaptation, distribution and reproduction in any medium or format, as long as you give appropriate credit to the original author(s) and the source, provide a link to the Creative Commons licence, and indicate if changes were made. The images or other third party material in this article are included in the article's Creative Commons licence, unless indicated otherwise in a credit line to the material. If material is not included in the article's Creative Commons licence and your intended use is not permitted by statutory regulation or exceeds the permitted use, you will need to obtain permission directly from the copyright holder. To view a copy of this licence, visit http://creativecommons.org/licenses/by/4.0/ The Creative Commons Public Domain Dedication waiver (http://creativecommons.org/publicdomain/zero/1.0/) applies to the data made available in this article, unless otherwise stated in a credit line to the data. 
Campylobacter infection involved antibiotics, especially in poultry industry. There is an urgency to develop alternative approaches due to a gradual increase in antibiotic-resistant Campylobacter [4]. It is also necessary to illustrate the mechanisms underlying the functions of such alternatives to support their development and implementation.

Research has increasingly shown that lactic acid bacteria (LAB) colonise the human gastrointestinal tract and play a vital role in maintaining intestinal function and well being of the host. Among the beneficial effects of LAB are bacteriostatic activities targeting pathogens such as Escherichia coli (E. coli), Salmonella and Listeria monocytogenes $[5,6]$. LAB have been shown to protect against Campylobacter infection. Nishiyama reported that LAB inhibited Campylobacter and colonisation of this pathogen was reduced by Lactobacillus gasseri SBT2055 isolated from healthy chicken [7]. Another study reported a decrease in $C$. jejuni invasion in the gut of turkey poults after treatment with Lactobacillus salivarius NRRL B-30514, suggesting that competitive exclusion can play a role [8]. Wagner et al. conducted a simulation experiment with immunodeficient and immunocompetent mice, they found that bifidobacteria and lactobacilli can increase colonisation resistance. Specifically, the results showed that bifidobacteria and lactobacilli can resist $C$. jejuni enteric persistence in the human gut [9]. To date, studies of antagonism against $C$. jejuni by LAB have mainly been conducted in vitro or in vivo. Unfortunately, some of the strains of LAB that showed a good ability to inhibit the growth of $C$. jejuni in vitro are less effective in antagonising $C$. jejuni in animals. Whether poultry or mice, all animal models have the disadvantages of high cost, a long cycle, cumbersome operation and insignificant immune response indicators, and do not permit rapid large-scale screening for LAB capable of effective antagonising $C$. jejuni.

The small, free-living (non-parasitic) soil worm Caenorhabditis elegans (C. elegans) has been widely used in biological studies as in vivo model, due to its short generation time, diminutive form and clear genetic background. To date, C. elegans has been used to study the effect of pathogenic microorganism, such as Salmonella enterica, Pseudomonas aeruginosa, Enterococcus faecalis and Staphylococcus aureus [10-14]. C. elegans is also increasingly used to screen for antimicrobials and probiotics [15]. In addition, institutional studies of the effects of LAB can be processed using C. elegans. However, no research on the use of $C$. elegans to screen for probiotic candidates antagonistic to $C$. jejuni has been conducted to date. Moreover, the molecular mechanisms underlying the protective mechanism of LAB have yet to be established. In this study, a C. elegans life-span experimental model was developed to investigate the response of nematodes to $C$. jejuni infection, enabling rapid evaluation of the protective effects of LAB and understanding of microbe-host interactions. At the same time, in this article, mice (with acute ileitis induced by Toxoplasma gondii ( $T$. gondii) infection which abrogating the colonisation resistance) and chicken were also used to validate the C. elegans model individually [52].

\section{Results}

\section{C. jejuni intake shortened the lifespan of $C$. elegans}

The harm caused by foodborne pathogens to the Caenorhabditis elegans is usually reflected in its lifespan. An experimental lifespan model of C. elegans was used to measure its response to $C$. jejuni infection (Fig. S1). The OP50 strain of Escherichia coli was used as the food source as this normally sustains nematodes on reaching the L4 stage. As shown in Fig. 1A, approximately 50\% of the $C$. elegans transferred to the $C$. jejuni group died within 5 days, whereas $80 \%$ of the C. elegans fed with OP50 (OP50 group) were still alive after 8 days. All of the nematodes in the OP50 group died within 21 days, whereas this was 13 days for the $C$. jejuni group. When the nematodes were fed OP50 for 3 days before being treated with $C$. jejuni (OP50/C. jejuni group), they were more resistant to $C$. jejuni and nearly $50 \%$ were still alive on day 10. However, the lifespan of $C$. elegans was shorter in the OP50/C. jejuni group than in the OP50 group. In addition, the initial number of $C$. jejuni cells that were recovered from the nematodes in the OP50/C. jejuni group was lower than that recovered from the nematodes in the $C$. jejuni group (Fig. 1C). This may be due to the protective effect of OP50 and/or shorter time of $C$. jejuni intake. However, to allow available time for lactic acid bacteria (LAB) intervention, C. elegans at day 3 after the L4 stage was chosen for the lifespan assay. Although the OP50/C. jejuni group was less effective in infecting $C$. elegans with $C$. jejuni than the $C$. jejuni group, the lifespan of $C$. elegans was still considerably shortened compared with the OP50 group.

The body size of $C$. elegans directly reflects its growth and development, which are closely related to its energy intake. In addition to directly causing disease, pathogens may also affect the lifespan of $C$. elegans due to their inability to be metabolised by this nematode. To determine whether the lifespan of $C$. elegans was shortened due to the pathogenicity of $C$. jejuni or reduced caloric intake, the body size of $C$. elegans fed with $C$. jejuni was compared with that of $C$. elegans fed with OP50 (Fig. 1B). The nematodes in the OP50 $+C$. jejuni group were fed equal amounts of OP50 and $C$. jejuni concurrently at the L4 stage. For 8 days, the nematodes in the OP50 $+C$. jejuni and OP50/C. jejuni groups were nearly the same size as those in the OP50 group, which indicated that the three groups had almost the same caloric intake 


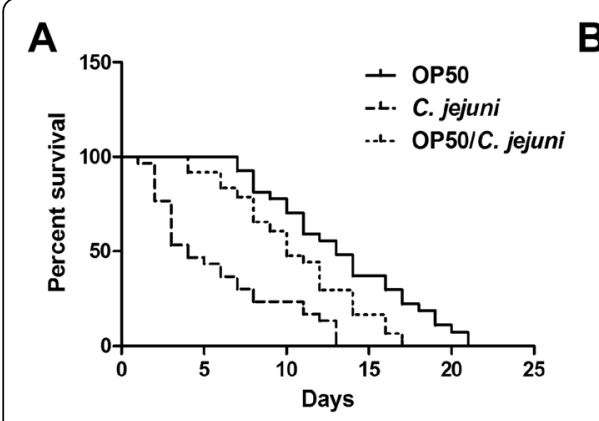

B

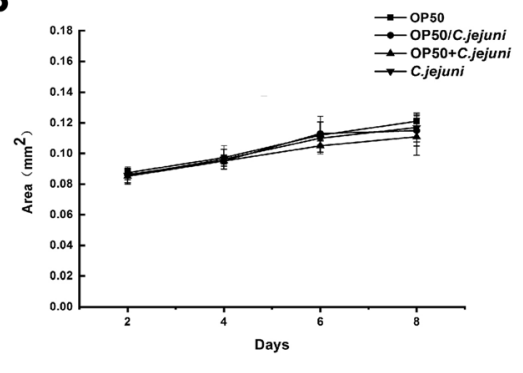

C

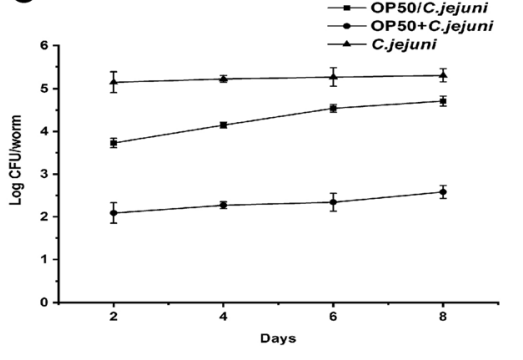

Fig. 1 Establishment of a life-span, body size and C. jejuni load in C. elegans assay. A Life-span of C. elegans treated with E. coli and C. jejuni in different ways. Day 0 marked the arrival of the nematodes at the $L 4$ stage before being fed thallus. B Body size of $C$. elegans treated with $E$. coli and C. jejuni in different ways. Day 0 marked the arrival of the nematodes at the L4 stage before being fed thallus. C C. jejuni load in C. elegans treated with E. coli and C. jejuni in different ways. Day 0 marked the point at which the nematodes were first fed C. jejuni

Table 1 Statistical analysis of the protection effects of LAB stains on C. elegans infected by C. jejuni NCTC $11168^{\mathrm{a}}$

\begin{tabular}{|c|c|c|c|c|c|c|c|}
\hline Groups $^{b}$ & Survival (\%) & DT50`(day) & $P$ & Groups $^{b}$ & Survival (\%) & DT50'(day) & $P$ \\
\hline OP50/C. jejuni & 20.16 & 6.83 & & ZX6/C. jejuni & 26.03 & 7.27 & 0.21 \\
\hline OP50 & 55.78 & 14.90 & $<0.01$ & PC-T7/C. jejuni & 25.56 & 8.85 & 0.21 \\
\hline 422/C. jejuni & 15.09 & 6.06 & 0.92 & JS-WX-9-1/C. jejuni & 29.01 & 9.58 & 0.20 \\
\hline B/C. jejuni & 18.02 & 7.38 & 0.88 & N8/C. jejuni & 28.67 & 8.09 & 0.18 \\
\hline G14/C. jejuni & 20.01 & 7.31 & 0.87 & 11,657/C. jejuni & 31.20 & 10.45 & 0.16 \\
\hline X14/C. jejuni & 16.42 & 7.23 & 0.88 & YM-1/C. jejuni & 31.29 & 11.13 & 0.11 \\
\hline LSQ3/C. jejuni & 19.03 & 7.06 & 0.87 & 676/C. jejuni & 27.05 & 10.66 & 0.10 \\
\hline 591/C. jejuni & 20.66 & 8.03 & 0.73 & JS-SZ-1-5/C. jejuni & 32.08 & 11.84 & 0.07 \\
\hline H17/C. jejuni & 20.32 & 6.79 & 0.58 & NCFM/C. jejuni & 36.89 & 12.49 & $<0.01$ \\
\hline LGG/C. jejuni & 21.05 & 7.65 & 0.57 & N34/C. jejuni & 37.52 & 12.39 & $<0.01$ \\
\hline 1101/C. jejuni & 21.03 & 7.55 & 0.57 & 427/C. jejuni & 38.02 & 13.06 & $<0.01$ \\
\hline N29/C. jejuni & 23.79 & 9.05 & 0.56 & X13/C. jejuni & 38.78 & 13.04 & $<0.01$ \\
\hline 408/C. jejuni & 21.43 & 8.41 & 0.55 & 720/C. jejuni & 39.11 & 12.21 & $<0.01$ \\
\hline H29M-8 M/C. jejuni & 22.17 & 7.81 & 0.55 & 2009/C. jejuni & 39.48 & 12.16 & $<0.01$ \\
\hline 730/C. jejuni & 21.89 & 6.91 & 0.53 & H33M-1/C. jejuni & 40.12 & 13.09 & $<0.01$ \\
\hline H9/C. jejuni & 22.81 & 8.45 & 0.51 & 13 M2/C. jejuni & 42.01 & 13.56 & $<0.01$ \\
\hline ZX7/C. jejuni & 22.95 & 9.18 & 0.46 & L103/C. jejuni & 44.41 & 12.67 & $<0.01$ \\
\hline 430/C. jejuni & 24.09 & 9.79 & 0.42 & G20/C. jejuni & 45.15 & 13.96 & $<0.01$ \\
\hline 675/C. jejuni & 22.67 & 7.98 & 0.41 & 1132/C. jejuni & 45.18 & 13.76 & $<0.01$ \\
\hline Z7/C. jejuni & 22.14 & 7.46 & 0.29 & H27-1 L/C. jejuni & 45.81 & 14.90 & $<0.01$ \\
\hline Z6/C. jejuni & 15.03 & 8.32 & 0.27 & 13-7/C. jejuni & 47.09 & 14.01 & $<0.01$ \\
\hline rui/C. jejuni & 25.81 & 10.46 & 0.26 & Z5/C. jejuni & 57.42 & 15.11 & $<0.01$ \\
\hline 9-5/C. jejuni & 29.58 & 9.21 & 0.25 & N9/C. jejuni & 43.28 & 13.70 & $<0.01$ \\
\hline
\end{tabular}

${ }^{a}$ Summary of two or more separate experiments. Survival of worms on the last day (day13) of the assays was estimated with the Kaplan-Meier survival analysis ${ }^{\mathrm{b}} \mathrm{OP} 50 / C$. jejuni: treatment with E. coli OP50 in the first 3 days and then with C.jejuni when C. elegans after L4 stage. OP50: treatment with E. coli OP50 during the whole experiment when C. elegans after $L 4$ stage. $L A B / C$. jejuni: treatment with $L A B$ in the first 3 days and then with C.jejuni when C. elegans after $L 4$ stage. The time of $\mathrm{L} 4$ stage nematodes before fed any thallus was considered 0 day

'DT50, the time at which half of the worms were dead 
during this period. Although the intestinal load of $C$. jejuni in the nematodes in the OP50/C. jejuni group was much larger than that in the OP50 $+C$. jejuni group, the load of C. jejuni in both groups remained stable (Fig. $1 C)$, indicating that the nematodes showed no preference for ingesting $C$. jejuni over $E$. coli. Therefore, substituting C. jejuni for E. coli did not lead to fasting in $C$. elegans, which would have affected its lifespan.

\section{LAB strains prolonged the lifespan of $C$. elegans infected}

\section{by $C$. jejuni}

The lifespan of C. elegans after bacterial infection is a common indicator of this nematode's antibacterial ability. To enable the rapid evaluation of the defensive effects of LAB, 44 LAB were assessed for their ability to protect $C$. elegans from $C$. jejuni infectionmediated death. On day 13 , the $C$. elegans that were only fed OP50 displayed a 50\% survival rate (DT50), whereas the DT50 of the C. elegans that were only fed C. jejuni was only $20 \%$. As shown in Table 1, the $\mathrm{LAB}$ isolates varied in their ability to protect the live nematodes, with survival rates ranging from 15 to $60 \%$. Among the tested isolates, some LAB provided high levels of protection, especially $13-7$, Z5 and N9 (each leading to a survival rate of $>40 \%$ ), and the lifespans of the nematodes in these three groups were significantly longer than the lifespan of the nematodes the OP50/C. jejuni group (Fig. 2).
LAB strains decreased the $C$. jejuni load in the intestine of C. elegans

Diminishing pathogenic bacterial colonisation in the intestinal tract is probably one of the underlying mechanisms of LAB in prolonging the lifespan of nematodes. In addition, LAB cells colonising the intestine of C. elegans play a role in decreasing the number of pathogenic bacteria. To investigate whether an association exists between the lifespan of $C$. elegans and the loadings of different bacteria in their intestines, the numbers of $L A B$, $C$. jejuni and E. coli OP50 in the intestines of C. elegans were counted. The $44 \mathrm{LAB}$ strains that showed various levels of protection were evaluated for their ability to persist in the intestines of $C$. elegans from day 2 to day 6. The loads of LAB on 13-7, Z5 and N9, which showed a strong ability to protect $C$. elegans against death, exceeded $\log 10^{4.5}$ colony-forming unit (CFU)/nematode during the assay (Fig. 3A and B). In contrast, the groups showed low levels of protection for the nematodes in the lifespan assay, and had the lowest colonisation levels of LAB (Table S2). The C. jejuni loads in C. elegans from day 2 to day 6 were also determined. As shown in Fig. $3 \mathrm{C}$ and D, the C. jejuni loads in C. elegans treated with 13-7, Z5 and N9 were almost 1.5 orders of magnitude lower than that in the OP50/C. jejuni group $\left(\log 10^{4.5}\right.$ $\mathrm{CFU} /$ nematode). In contrast, the groups offering low levels of protection in terms of lifespan did not significantly decrease the $C$. jejuni load (Table S3). There were no obvious differences in the OP50 load between the

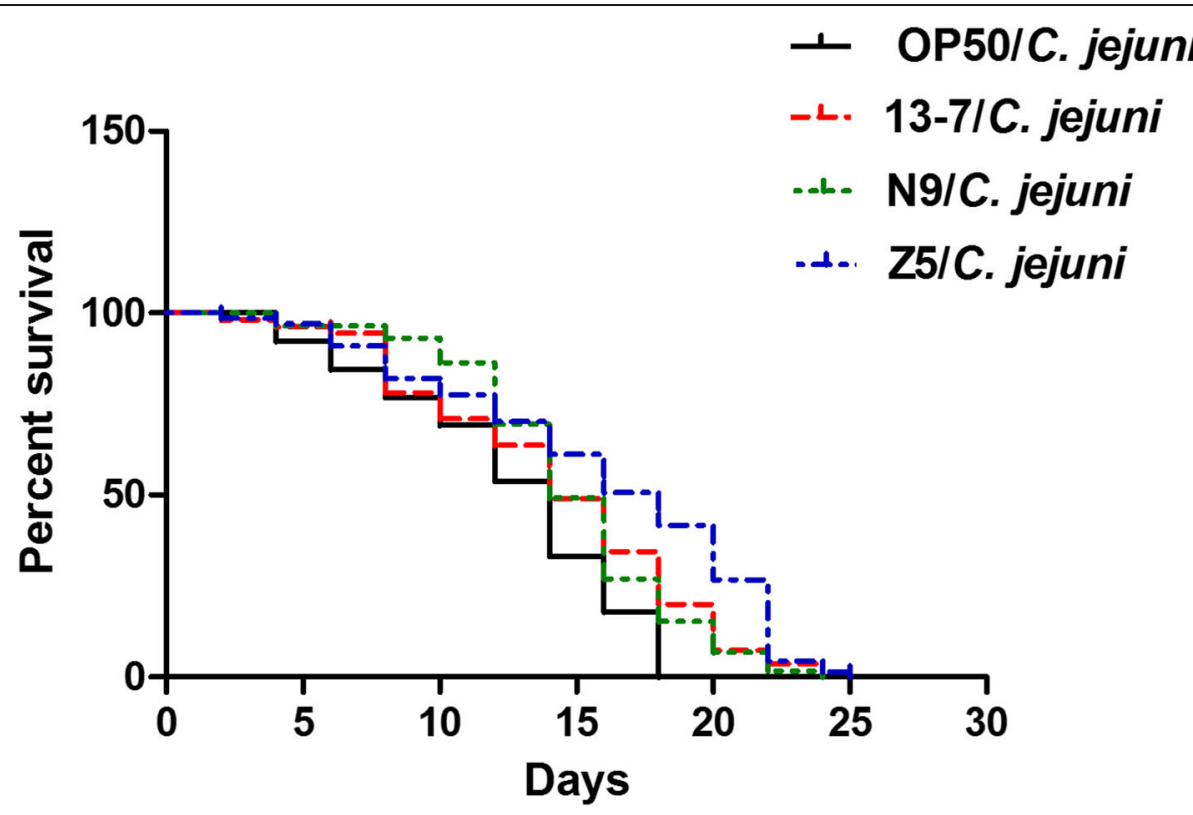

Fig. 2 Differential effects of $L A B$ on the survival of C. elegans infected with C. jejuni. OP50/C. jejuni: treated with E. coli OP50 for the first 3 days and then with $C$. jejuni after the $L 4$ stage. $L A B / C$. jejuni: treated with $L A B$ for the first 3 days and then with $C$. jejuni after the $L 4$ stage. Day 0 marked the arrival of the nematodes at the $L 4$ stage before being fed thallus 


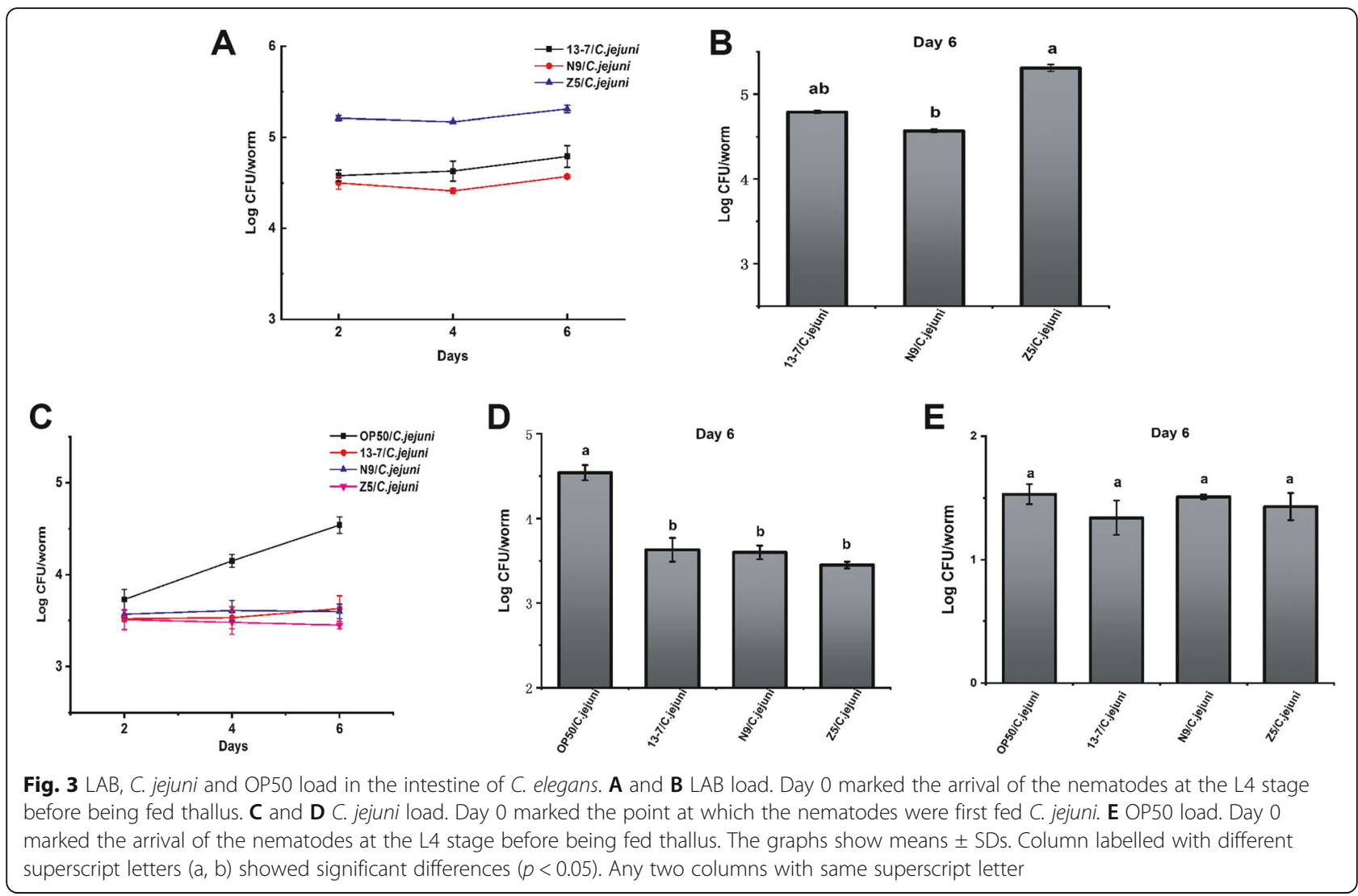

OP50/C. jejuni group and the other LAB intervention groups ( $\log 10^{1.2}-10^{1.5} \mathrm{CFU} /$ nematode) on day 6 (Fig. $3 \mathrm{E}$ and Table S4). The results of a correlation analysis showed that the LAB and C. jejuni loads were highly positively correlated and highly negatively correlated with the survival of $C$. elegans, respectively. In addition, the OP50 load was not correlated with the survival of $C$. elegans (Table 2).

No change on body size and pharyngeal pumping of $C$. elegans infected by $C$. jejuni under LAB intervention

As mentioned, the body size of C. elegans directly reflects its growth and development, which are closely related to its energy intake. In addition to body size, pharyngeal pumping represents the feeding capacity of C. elegans and is another key index for physiological

Table 2 Correlation analysis of LAB colonization and C.jejuni load in the intestine and survival of $C$. elegans

\begin{tabular}{|c|c|c|}
\hline \multicolumn{2}{|l|}{ Comparison Groups } & \multirow{2}{*}{$\begin{array}{l}\text { Pearson Correlation } \\
\mathrm{R}^{2}=0.517^{* *}\end{array}$} \\
\hline Colonization of LAB & Survival of C. elegans & \\
\hline C. jejuni load & Survival of C. elegans & $R^{2}=-0.615^{* *}$ \\
\hline Colonization of LAB & C. jejuni load & $R^{2}=-0.424^{*}$ \\
\hline OP50 load & Survival of C. elegans & $R^{2}=-0.013$ \\
\hline
\end{tabular}

* Indicates statistically significant differences at $p<0.05$

** Indicates statistically significant differences at $p<0.01$ activities. To determine whether the longevity effects of $\mathrm{LAB}$ were the result of caloric reduction, the body size (Table S5) and pharyngeal pumping rate (Table S6) of $C$. elegans fed with $44 \mathrm{LAB}$ strains and $C$. jejuni $(\mathrm{LAB} / C$. jejuni groups) were compared with those of the nematodes in the OP50/C. jejuni group. The nematodes in the OP50/C. jejuni group and those in the 13-7, Z5 and N9 intervention groups showed little difference in body size (Fig. 4A and B). Similarly, little difference was observed in pharyngeal pumping between the groups. Figure $4 \mathrm{C}$ showed that the pharyngeal pumping rate ranged from 50 to 58 per 30 s in the OP50/C. jejuni group and $13-7, \mathrm{Z} 5$ and N9 intervention groups. There were no significant differences in body size or pharyngeal pumping of nematodes treated by these LAB, thus demonstrating the varied longevity of $C$. elegans treated with $C$. jejuni.

\section{Influence on C. jejuni growth by co-culturing with E. coli OP50}

To investigate whether the substances produced by $E$. coli OP50, such as certain bacteriocins, could kill $C$. jejuni, the viability of $C$. jejuni cultured with and without live E. coli OP50 was measured. As shown in Fig. 5, the number of $C$. jejuni increased in both tests after $24 \mathrm{~h}$ of incubation and did not exhibit a significant difference 

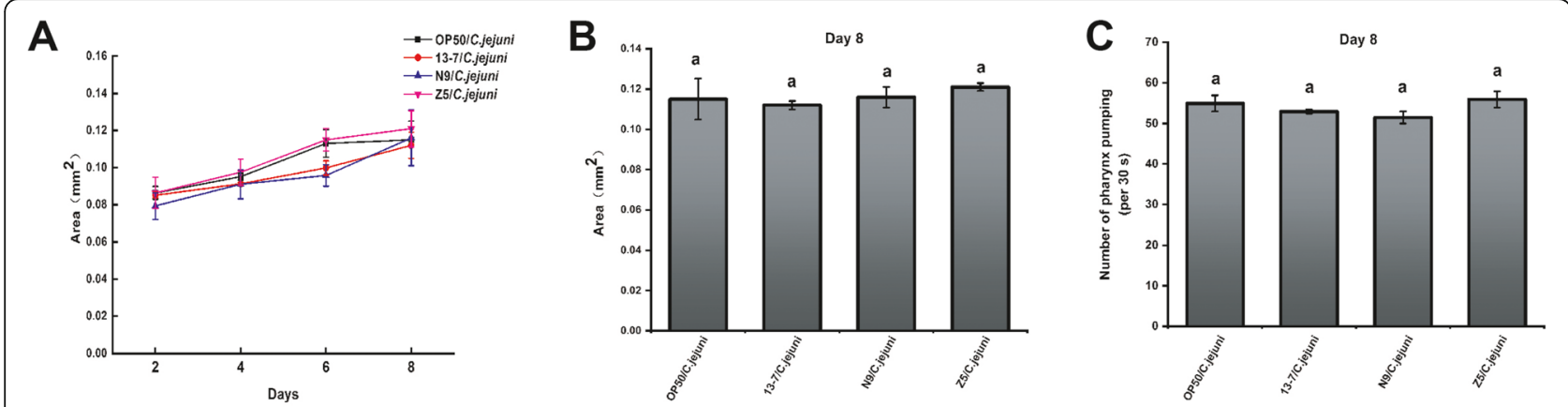

Fig. 4 Effects of $L A B$ on body size and pharynx pumping of C. elegans. A and B Body size (area) of C. elegans treated with $L A B$ and $C$. jejuni on day 8. Day 0 marked the arrival of the nematodes at the $L 4$ stage before being fed thallus. $C$ Effects of $L A B$ on the pharynx pumping of $C$. elegans infected by C. jejuni. Pharynx pumping (per 30s) of C. elegans treated with LAB and C. jejuni on day 8 . All day 0 marked the arrival of the nematodes at the $L 4$ stage before being fed thallus. The graphs show means \pm SDs. Column labelled with different superscript letters ( $a$, b) showed significant differences $(p<0.05)$. Any two columns with same superscript letter

between the tests. Furthermore, the number of $C$. jejuni was uninfluenced after the live E. coli OP50 were added to the growing $C$. jejuni after $48 \mathrm{~h}$ of incubation.

\section{LAB strains upregulated the immune gene transcription}

Regulation of the host's immune system through pivotal signalling pathways is an underlying mechanism by which LAB can prolong the lifespan of a nematode and protect it against pathogen infection. To determine the mechanism by which LAB protected $C$. elegans against
C. jejuni infection, the transcription levels of 14 immune genes (tir-1, nsy-1, sek-1, pmk-1, spp-1, clec-85, abf-2, clec-60, lys-7, daf-16, age-1, dbl-1, skn-1 and bar-1) of C. elegans on day 6 were compared between the OP50, $\mathrm{OP} 50 / C$. jejuni, and $\mathrm{LAB} / C$. jejuni groups. As shown in Fig. $6 \mathrm{~A}, \mathrm{~B}$ and $\mathrm{C}$, the transcription of tir-1 and pmk-1 (MAPK signalling pathway genes) and bar-1 (an antioxidant gene) increased to some extent when $C$. elegans was infected with $C$. jejuni. In addition, slight increases were observed in some of the defence immune genes of

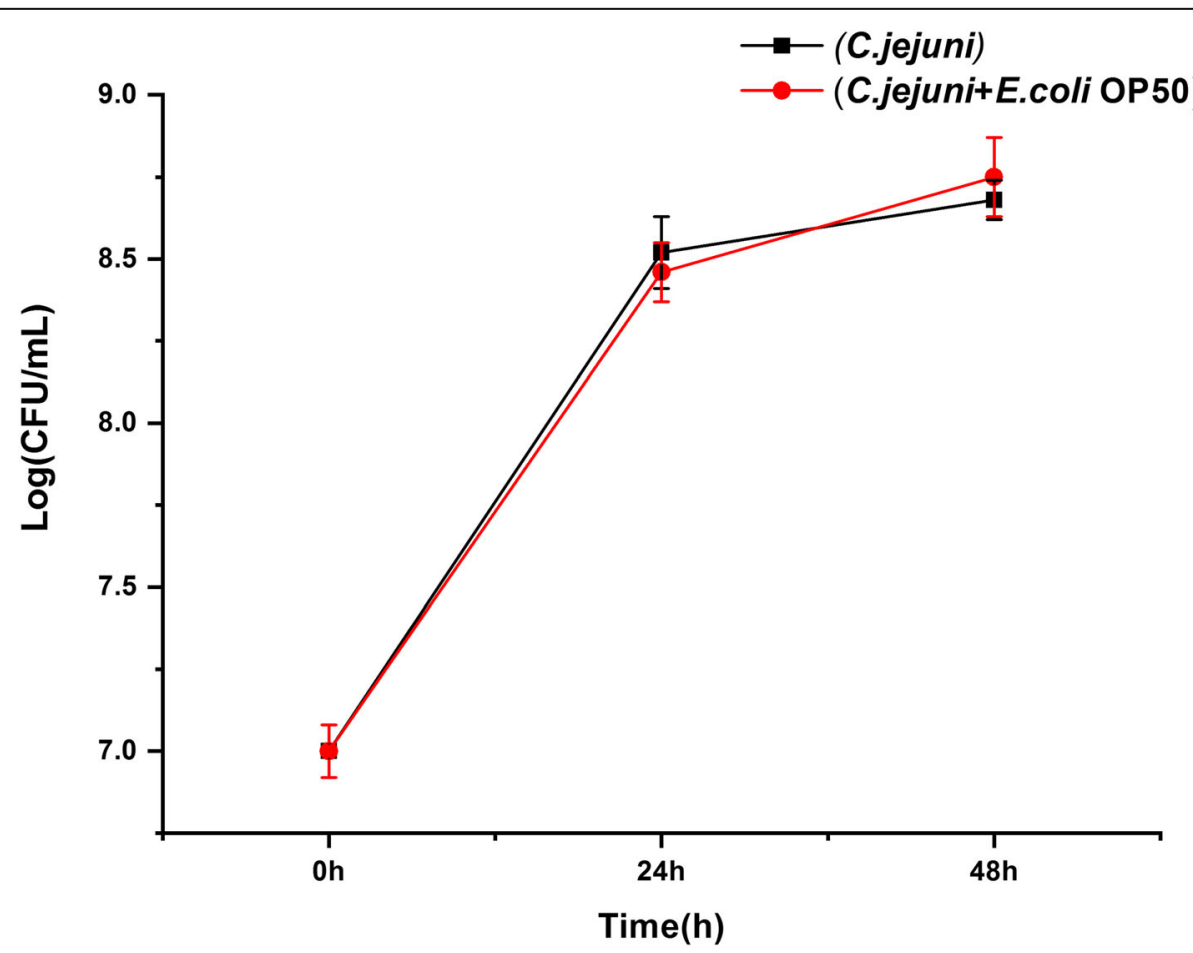

Fig. 5 Live cell counts of C. jejuni in the co-culture with E.coli OP50 strain 


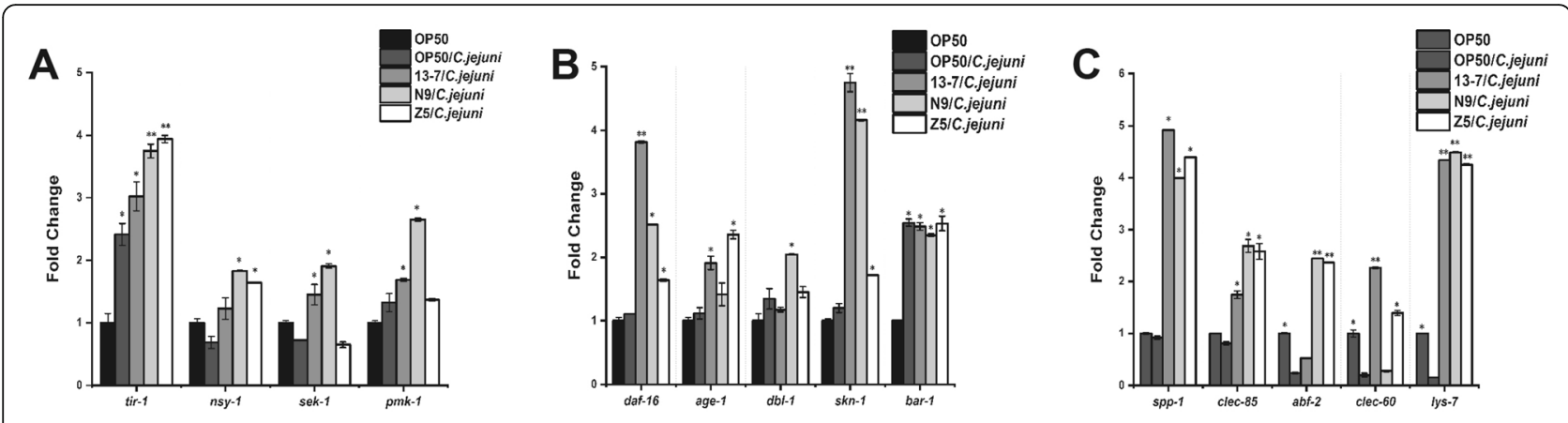

Fig. 6 Differential effects of $L A B$ on transcription of immune genes of $C$. elegans on day 6 . Day 0 marked the arrival of the nematodes at the $L 4$ stage before being fed thallus. ${ }^{*}$ Indicates statistically significant differences at $p<0.05 .{ }^{* *}$ Indicates statistically significant differences at $p<0.01$

C. elegans infected with C. jejuni, such as daf-16 and age-1 (Daf-16 signalling pathway genes) and dbl-1 (a TGF- $\beta$ signalling pathway gene).

The 14 immune genes of C. elegans were also examined after treatment with 44 LAB strains, which revealed a variation in the protection against $C$. jejuni-induced nematode mortality (Table S7). It was found that the expression level of the immune genes of $C$. elegans did not change significantly after LAB intervention without $C$. jejuni infection, indicating that the LAB strains were safe for a healthy host (Table S8). As shown in Fig. 6A, B and $C$, the treatments of nematodes with 13-7, N9 and Z5 significantly enhanced the transcription of the MAPK signalling pathway genes $n s y-1$, sek-1 and $p m k-1$, the antioxidant gene $s k n-1$, and the Daf-16 signalling pathway genes age-1 and daf-16. The transcription levels of the antibacterial peptide genes spp-1, clec-85 and lys-7 in these $\mathrm{LAB}$ intervention groups were 3-4 times higher than those in the group infected with $C$. jejuni alone. These data suggest that the ability of certain LAB to protect nematodes against $C$. jejuni-induced mortality was correlated with their influence on the transcription levels of immune genes. In contrast, when C. elegans was treated with LAB offering low levels of protection for survival, the transcription levels of their defence genes were almost identical to those of C. elegans infected with C. jejuni alone (Table S7).

\section{LAB strains that prolonged the lifespan of $C$. elegans decreased the $C$. jejuni load in mice with $T$. gondii- induced acute ileitis}

The LAB that protect nematodes against bacterial infection might be inapplicable to mammals due to the obvious differences between the organisms. To investigate whether the LAB screened from $C$. elegans had the same effects in mammals, 11 LAB strains (including 13-7, Z5 and N9) with different effects on the immune gene transcription of C. elegans were further investigated to determine their abilities to decrease the $C$. jejuni load in mice. Toxoplasma gondii was used to induce acute ileitis to avoid colonisation resistance in normal mice. Seven days after T. gondii infection, the mice developed acute ileitis and most died; thus, the $C$. jejuni loads were determined on day 9. The $C$. jejuni load in the faeces of mice in the C. jejuni-infected group reached $10^{8} \mathrm{CFU} / \mathrm{g}$ (Fig. 7A). The LAB strains that showed the strongest
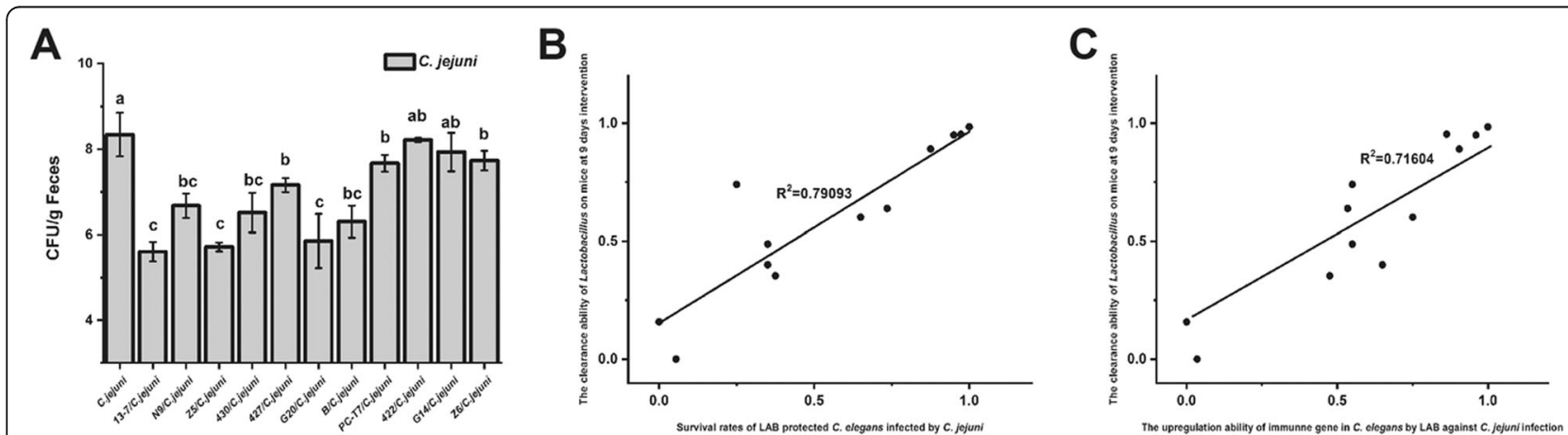

Fig. 7 Differential effects of $\angle A B$ on C. jejuni load in mice infected by C. jejuni and correlation analysis between C. elegans and mice models. A Culturable C. jejuni in mice faeces on day 9 . The graphs show means \pm SDs. Column labelled with different superscript letters $(a, b, c)$ showed significant differences $(p<0.05)$. Any two columns with same superscript letter. B Correlation analysis of ability of $L A B$ strains to clear $C$. jejuni in infected mice on day 5 and the survival rate of $C$. jejuni-infected nematodes under $L A B$ treatment. $C$ Correlation analysis of ability of $L A B$ strains to clear $C$. jejuni in infected mice on day 9 and upregulate immune gene transcription to protect $C$. elegans against C. jejuni infection 
ability to protect C. elegans (i.e. 13-7, Z5 and G20) also exerted a superior suppressive effect on the $C$. jejuni load $\left(<10^{6} \mathrm{CFU} / \mathrm{g}\right.$ of faeces) in the intestinal tract of mice. Strains 422 and G14, which showed poor protective effects in terms of the lifespan of C. elegans, correspondingly played an inconspicuous role in suppressing the C. jejuni load ( $10^{8} \mathrm{CFU} / \mathrm{g}$ of faeces). Consistently with this, PC-T7 and Z6, which had previously offered moderate and low levels of protection, respectively, to $C$. elegans, showed slight suppressive effects on the $C$. jejuni load $\left(10^{7}-10^{8} \mathrm{CFU} / \mathrm{g}\right.$ of faeces). Meanwhile, N9 and 430, which had previously offered high and moderate levels of protection to C. elegans, respectively, had moderate suppressive effects on the $C$. jejuni load $\left(10^{6}-\right.$ $10^{7} \mathrm{CFU} / \mathrm{g}$ of faeces).

A correlation analysis was conducted to examine the relationship between the ability of the 11 LAB strains to remove $C$. jejuni in mice and the survival rate of $C$. jejuni-infected nematodes or the uprefulation ability of immune gene (spp-1) in C. elegans by LAB against $C$. jejuni infection. The correlation coefficient $\mathrm{R}^{2}$ reached 0.79093 and 0.71604 , indicating that the C. jejuni-antagonistic activity of LAB in C. elegans by upregulating worms immune gene transcription to prolong life was significantly correlated with the $C$. jejuni-antagonistic activity of LAB in mice (Fig. 7B and C).

\section{LAB strains that prolonged the lifespan of $C$. elegans} decreased the $C$. jejuni load in chickens

Outbreaks of campylobacteriosis can occur if humans ingest undercooked poultry contaminated with $C$. jejuni. Studies have shown that LAB applied to fodder could reduce Campylobacter colonisation in poultry and stop the outbreak of this disease at its source. To investigate whether the LAB screened from $C$. elegans had the same effects in poultry, $11 \mathrm{LAB}$ strains with different effects on immune gene transcription in C. elegans were investigated to determine their abilities to remove $C$. jejuni in chickens. The inhibitory effect of LAB on $C$. jejuni colonisation in the cecum of chickens was examined. Approximately $24 \mathrm{~h}$ after hatching, chickens were inoculated orally with $C$. jejuni, and then LAB were administered daily for 2 weeks. The CFU count of $C$. jejuni in the cecum of chickens in all groups was evaluated on day 23. The average value of C. jejuni increased to $10^{8}$ $\mathrm{CFU} / \mathrm{g}$ of cecal content in the $C$. jejuni-infected group (Fig. 8A). Strains Z5 exerted significant suppressive effect on C. jejuni colonisation in the cecum of chickens, which resulted in the reduction of the $C$. jejuni load to below $10^{4} \mathrm{CFU} / \mathrm{g}$ of cecal content in this group. Meanwhile, strains $430, B$ and G14 showed poor abilities to remove $C$. jejuni from the cecum of chickens. The $C$. jejuni loads in these groups were $>10^{7} \mathrm{CFU} / \mathrm{g}$ of the cecal content. The effects of these three strains in eliminating C. jejuni in C. elegans were similar to their effects in eliminating $C$. jejuni in chickens. However, strains 430 and B exhibited moderate $C$. jejuni antagonism in C. elegans, which were stronger than their performance in chickens. In addition, 13-7, N9, G20, PCT7, 422 and Z6 showed moderate ability to eliminate $C$. jejuni in the cecal content of chickens, with a $C$. jejuni load of $10^{4.8}-10^{5.5} \mathrm{CFU} / \mathrm{g}$ of faeces. Two of those strains (422 and Z6) presented a certain degree of inconsistency in the $C$. jejuni antagonism in chickens and nematodes.

A correlation analysis was conducted to examine the relationship between the ability of the 11 LAB strains to remove $C$. jejuni in chickens and the survival rate of $C$. jejuni-infected nematodes or the uprefulation ability of immune gene (spp-1) in C. elegans by LAB against $C$. jejuni infection. The $\mathrm{R}^{2}$ value reached 0.50071 and 0.63811 , indicating that the C. jejuni-antagonistic activity of LAB in C. elegans by upregulating worms immune
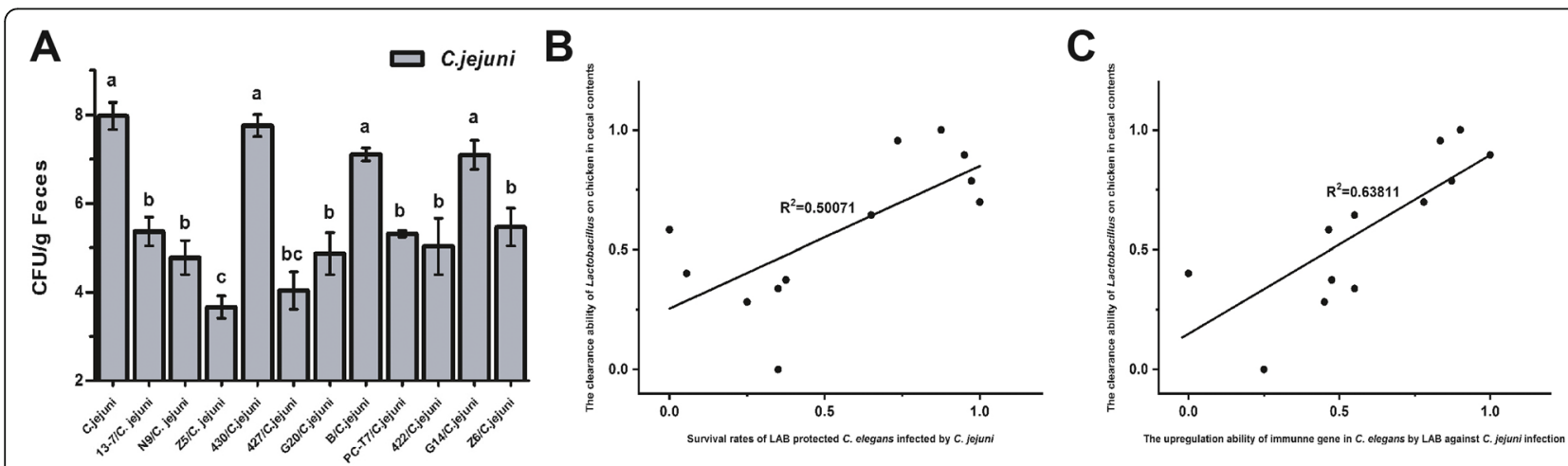

Fig. 8 Differential effects of $L A B$ on C. jejuni load in chicken infected by C. jejuni. A Culturable C. jejuni in chicks' cecal contents on day 23. The graphs show means \pm SDs. Column labelled with different superscript letters $(a, b, c)$ showed significant differences $(p<0.05)$. Any two columns with same superscript letter. B Correlation analysis of ability of $L A B$ strains to clear $C$. jejuni in infected chicks' cecal contents and the survival rate of $C$. jejuni-infected nematodes under $L A B$ treatment. C Correlation analysis of ability of $L A B$ strains to clear $C$. jejuni in infected chicks' cecal contents and upregulate immune gene transcription to protect $C$. elegans against $C$. jejuni infection 
gene transcription to prolong life was correlated with their C. jejuni-antagonistic activity in chickens (Fig. 8B and $\mathrm{C})$.

\section{Discussion}

The model organism C. elegans has been treated with numerous pathogenic microorganisms from animals and humans to examine the relationships between pathogenic bacteria and their hosts [10-14]. In this study, a lifespan experimental model of C. elegans was established to examine the response of this nematode to $C$. jejuni infection. Our experiments revealed that LAB strains varied in their ability to defend C. elegans against infection from $C$. jejuni (NCTC 11168). In addition, the antagonistic effects of LAB isolates on $C$. jejuni in C. elegans were verified in both mice and chickens. The lifespan experimental model of $C$. elegans not only provided a useful method of screening for LAB candidates with the potential to mitigate $C$. jejuni infection, but also helped to explain the defence mechanism imparted by the $\mathrm{LAB}$ isolates. The findings also indicated that LAB defended $C$. elegans not only by inhibiting $C$. jejuni colonisation in the intestines, but also by activating the defence immune genes of C. elegans.

The natural physiological activities of nematodes are usually halted by the colonisation of invading pathogens [17-19]. Some LAB, such as Lacticaseibacillus rhamnosus [20], Lactobacillus acidophilus [21], Lactobacillus fermentum [13], Lactobacillus gasseri and Lactobacillus plantarum [22], have been reported to show high shielding efficiencies to nematodes from pathogens, to diminish the risk of bacterial colonisation in the intestinal tract, and to prolong the lifespan of nematodes. The intestinal colonisation of $C$. jejuni may occur in humans and animals, and is often observed in poultry. The binding of fimbrial adhesins to the host's intestinal tract is a precursor to $C$. jejuni nosogenesis, which leads to diseases such as diarrhoea [23, 24]. Many studies have shown that $C$. jejuni colonisation and growth in the host's intestinal tract can be reduced by some LAB, such as Bifidobacterium and Lactobacillus salivarius [25, 26]. The results of the present study confirmed that the level of $C$. jejuni in the intestinal tract of $C$. elegans was affected differentially by LAB strains, which offered strong to weak levels of protection. The C. jejuni load in C. elegans was strongly correlated with its lifespan, which indicated that the inhibition of $C$. jejuni colonisation in the intestinal tract was one of the mechanisms through which LAB offered protection to C. elegans. As reported, some foodborne pathogens, such as Salmonella enterica serovar Typhimurium [21] and entero-invasive E. coli [27], can colonise the intestinal tract of nematodes to form a persistent lethal infection. The colonised LAB in the intestine continuously produce antibacterial products, and can also occupy the adhesion sites of pathogens, which jointly exert antibacterial effects [54]. However, in this study, the LAB load and C. jejuni load in $C$. elegans were negatively correlated, indicating that some LAB may alleviate the damage caused by infected pathogens in other ways, such as through the regulation of immune gene expression in nematodes.

In non-mammalian taxa, mice and some primates, calorie restriction is normally used to increase longevity and ease the consequences of ageing [28-30]. Researchers have also investigated the caloric intake of $C$. elegans after LAB intervention through measurements of body size and the pharyngeal pumping rate [10-14]. But in this article, under the intervention of LAB, there was no relationship between $C$. jejuni- infected $C$. elegans lifespan extension and its morphological or pharyngeal pumping.

With the growth and reproduction of LAB in a host's intestine, the immune system is regulated through pivotal signalling pathways with LAB involvement [31]. The host's immune response to pathogens may be induced by $L A B$ [32]. In addition, LAB strains with protective efficacy, such as L. rhamnosus GG [33], L. plantarum [34] and Lactobacillus delbrueckii [35], can enhance the host's immune defence against E. coli, S. typhimurium and Streptococcus pyogenes infection by exciting the MAPK signalling pathway and through toll-like receptors. Moreover, LAB may defend the host by adjusting gene expression through cytokine and chemokine activity $[36,37]$. Another possible mechanism is the enhancement of the membrane barrier [38, 39]. Caenorhabditis elegans has two main signalling pathways: P38-MAPK and TGF- $\beta$. Its specific defence system, including antimicrobial responses, falls short of an adaptive immune system [40]. Nevertheless, the upregulation of MAPK pathway gene (tir-1, nys-1, sek-1 and pmk-1) expression can enable $C$. elegans to resist various microbial infections. These findings demonstrated the activation of the MAPK pathway through LAB intervention [31]. In the present study, the LAB strains offered good levels of protection for C. elegans against C. jejuni infection, and significantly enhanced the transcription of the MAPK pathway genes. As a downstream molecule regulated by the MAPK signalling pathway, the fork-head family transcription factor DAF-16 has been shown to adjust gene expression to improve dauer formation in the larval phase of nematodes, and to increase resistance and longevity when mature [4143]. The results of the present study were consistent with the above findings, suggesting that the studied LAB strains prolonged the lifespan of C. elegans by upregulating DAF-16 via the MAPK signalling pathway. 
In addition, it is considered that the cluster of C-type lectins can mask bacterial attachment and enhance resistance to microbial infection [44]. The increased levels of clec-60, clec-85, spp-1 and abf-2 upon LAB pretreatment in this study also indicate the ability of some LAB to protect nematodes against $C$. jejuni infection. $S k n-1$ and bar-1 may also play critical roles in defending a host and prolonging its survival. The increased level of $s k n-1$ in pre-treated nematodes has been found to be due to antioxidant defence followed by their improved survival [45-47]. Partly consistent with this claim, skn-1 transcription in the current study was significantly enhanced by the LAB strains that prolonged the lifespan of C. elegans. Although the TGF- $\beta$ pathway is a major defence system in C. elegans, $d b l-1$ showed almost no obvious changes in the transcription level across the LAB intervention groups, indicating that this gene may not play an essential role in protecting against $C$. jejuni infection. Moreover, the immune genes of C. elegans without $C$. jejuni infection did not change significantly after intervention by the LAB strains. The studied LAB only played a significant role when the nematodes received external infection, suggesting that $\mathrm{LAB}$ are safe for a healthy host.

As the growth temperature of $C$. elegans is much lower than the temperature in the human gut, and the microbiota of $C$. elegans is much simpler than that of a mammal, the reliability of this lifespan model of $C$. elegans in testing the efficiency of LAB antagonism against C. jejuni colonisation in mammals should be confirmed. In addition, because the short cycle of acute infection in mice represents a research limitation, chickens were used to investigate the long-term effects in this study. Nevertheless, the $C$. jejuni-infected chicken model has been widely used in screening probiotics for feed with $C$. jejuni antagonism, which could be applied as antibiotic substitutes [16]. The significant correlation between the ability of LAB to remove $C$. jejuni in mice and chicken and their ability to upregulate immune gene transcription to protect $C$. elegans against $C$. jejuni infection indicates that the lifespan model of $C$. elegans infected with C. jejuni can be applied to some extent to C. jejuni-infected mammals and birds. Although the correlation between the different models was relatively good, it was undeniable that some strains had different antibacterial effects in these models. The reason for the difference may be physiological differences (such as body temperature) on different hosts or different growth characteristics of LAB in the body (the ability of colonization or production of antibacterial substances). The results indicate that $C$. elegans is a good model organism for screening $C$. jejuni-resistive LAB strains on a large scale.

In this study, the observed effects of LAB strains on the antagonistic ability of C. jejuni infection in nematodes with its genome determinants were also investigated. Taking L. plantarum N9 as an example, a glycosyltransferase-encoding gene that differed to that of other L. plantarum strains was found in COG and NR annotations. This gene regulates the synthesis of extracellular polysaccharides in bacterial strains. Exopolysaccharides have been proven to have a series of physiological health care effects, such as antioxidant properties, immune regulation and resistance to pathogenic bacteria. Therefore, the analysis results suggest that the core functional genes of LAB endow them with the ability to alleviate the infection of $C$. jejuni in nematodes.

\section{Conclusion}

This study established a lifespan model of C. elegans that can measure the response of this nematode to $C$. jejuni. Different LAB species exhibited different effects on the response of $C$. elegans to infection with $C$. jejuni. The inhibition of intestinal colonisation by $C$. jejuni may have been one of the mechanisms through which LAB protected C. elegans, and the protection offered by LAB may also have derived partly from their activation of the defence immune genes of $C$. elegans. This lifespan model of C. elegans can be used to screen for C. jejuni-antagonistic LAB on a large scale.

\section{Methods}

\section{C. elegans, $\mathrm{LAB}$ and $\mathrm{C}$. jejuni}

C. elegans N2 Bristol wild-type strain and E. coli OP50 (Caenorhabditis Genetics Center, Minnepolis, University of Minnesota) was used in the study. $20^{\circ} \mathrm{C}$ was used for nematodes maintence and cultivation. The procedures for the cultivation, maintenance and synchronization of the nematode have been reported $[48,55]$. Before using E. coli OP50 on every experiment, its concentration was adjusted to $1 \times 10^{8}$ colony-forming units $(\mathrm{CFU}) / \mathrm{mL}$ in sterile phosphate-buffered saline solution (PBS).

Six LAB were purchased from the American Type Culture Collection (ATCC) or the Japan Collection of Microorganisms and 38 were isolated from human faeces from different habitats and traditional fermented food. The selected LAB were identified through $16 \mathrm{~S}$ gene sequencing. All the isolates were deposited at the Culture Collection of Food Microorganisms at Jiangnan University (Table S1). The whole genome sequence of 12 isolates, namely N8, N9, 422, 427, 430, Z5, L103, X13, JSSZ-1-5, JS-WX-9-1, 9-5 and H27-1 L were aligned and identified as new strains of the corresponding species reported. The other 26 isolates are assumed tentative strains, for they were isolated from samples of different origin, geographical location and span over seven years. All of the LAB were cultivated in deMan, Rogosa and Sharpe (MRS) agar at a $2 \%(\mathrm{v} / \mathrm{v})$ inoculum size at $37^{\circ} \mathrm{C}$ 
for 18 to $20 \mathrm{~h}$ with an atmosphere of $85 \% \mathrm{~N}_{2}, 10 \% \mathrm{CO}_{2}$, and $5 \% \mathrm{H}_{2}$. Before using LAB on every experiment, its concentration was adjusted to $1 \times 10^{9}$ colony-forming units $(\mathrm{CFU}) / \mathrm{mL}$ in sterile phosphate-buffered saline solution (PBS).

The source and cultivation of C. jejuni NCTC 11168 have been reported [55]. Before using $C$. jejuni on every experiment, its concentration was adjusted to $1 \times 10^{9}$ colony-forming units $(\mathrm{CFU}) / \mathrm{mL}$ in sterile phosphatebuffered saline solution (PBS).

\section{Life-span experimental analysis of $C$. elegans}

The nematodes were synchronised as previously described [27]. To build a model for the experimental analysis of worm death induced by C. jejuni, $10^{9} \mathrm{CFU} / \mathrm{mL}$ of fresh and live $C$. jejuni was prepared for worms every day during the whole experiment. Each group had 80100 worms at L4 stage and three independent replications were performed for each assay. As shown in Fig. S1, after L4 stage, worms fed only with E. coli OP50 formed the negative control group (OP50 group). The worms fed only with $C$. jejuni was called $C$. jejuni group. Throughout the experiment, OP $50+C$. jejuni group was the nematodes fed by $E$. coli OP50 and C. jejuni at the same time after L4 stage. In an experiment undertaken to evaluate the role of $\mathrm{LAB}$ in protecting the worms against death caused by $C$. jejuni, the worms were either fed $10^{8} \mathrm{CFU} / \mathrm{mL}$ E. coli OP50 or $10^{9} \mathrm{CFU} /$ $\mathrm{mL}$ LAB strains for the first 3 days. After $72 \mathrm{~h}$ of incubation, the worms were moved to new $6 \mathrm{~cm}$ plates with $C$. jejuni at a concentration of $10^{9} \mathrm{CFU} / \mathrm{mL}$. The worms that were first fed E. coli OP50 $(72 \mathrm{~h})$ and next $C$. jejuni formed the $C$. jejuni reference group (OP50/C. jejuni group); those treated with LAB (72 h) before $C$. jejuni were regarded as the $\mathrm{LAB}$ protection groups ( $\mathrm{LAB} / \mathrm{C}$. jejuni group). A worm was considered dead when it failed to respond to gentle touch with a worm picker. The numbers of live worms were recorded, and the probability of their survival was calculated as described previously [27].

\section{Examination of bacterial load in the intestine of $C$. elegans}

The numbers of E. coli OP50, Lactobacillus and C. jejuni in the nematodes' intestine were determined with some modification of the method described previously [27]. Examination of Lactobacillus was executed on $\mathrm{LAB} / C$. jejuni groups. The sampling ( 50 worms per sample) was done every 2 days and day 0 marked the arrival of the nematodes at the L4 stage before being fed thallus. At the same time, on day 6, E.coli OP50 load in all groups was detected. $C$. jejuni test also followed the same method on Lactobacillus. But, day 0 marked the point at which the nematodes were first fed C. jejuni. After surface sterilization, the worms were mashed mechanically with a pellet pestle motor, re-suspended in the M9 buffer, and inoculated onto eosin-methylene blue medium (EMB medium), MRS or Columbia blood agar for counting of E. coli OP50, Lactobacillus and C. jejuni, respectively. At least three independent replications were performed for each assay.

\section{Measurement of body size and pharynx pumping}

The live worms were examined for their body size measurements every 2 days and their pharynx pumping on the eighth day after they were fed thallus at the L4 stage. Images of adult nematodes were taken with a VCTVBIT digital microscope (Shimadzu, Kyoto, Japan) and analyzed using the ImageJ software. In this system, the area of the worm's projection was estimated automatically and used as an index of body size. And the worm's pharynx pumping was measured per 30s.

\section{Evaluation of the effects on the growth of $C$. jejuni by co- culturing E. coli OP50}

The growth of $C$. jejuni co-cultured with E. coli OP50 was determined by the following method as previously described [53]. The C. jejuni cells $\left(10^{7} \mathrm{CFU} / \mathrm{mL}\right)$ suspended in antibiotic-free brain heart infusion broth (BHIB) containing 5\% serum were incubated under microaerophilic conditions for $48 \mathrm{~h}$ at $37^{\circ} \mathrm{C}$ in the presence of a $10 \%$ volume of live E. coli OP50 (10 $7 \mathrm{CFU} /$ $\mathrm{mL})$. Both were incubated together in liquid medium. The viability of $C$. jejuni was evaluated from the number of viable CFUs in C. jejuni culture as described above on C. jejuni-selective plates. At least three independent replications were performed for this assay.

\section{RNA extraction, reverse transcription and quantitative real-time PCR analysis}

The whole RNA of C. elegans and of the bacteria used in the life-span experiment was extracted. About 100 worms were prepared for the lysates. The RNA was extracted as previously described [27]. Total RNA of bacteria and $C$. elegans from the life span assays was extracted from lysates of the worms, using the mirVana miRNA Isolation Kit (Ambion, TX) according to manufacturer's instructions.

The transcription of mitogen-activated protein kinase (MAPK) pathway genes (tir-1,nsy-1, sek-1 and pmk-1), antimicrobial peptide genes (spp-1, clec-85, abf-2, clec-60 and lys-7), Daf-16 pathway genes (daf-16 and age-1), a TGF- $\beta$ pathway gene $(d b l-1)$ and antioxidant genes $(s k n-$ 1 and bar-1) in C. elegans was determined by quantitative polymerase chain reaction (qPCR) [49]. SuperScript first-strand synthesis system (Invitrogen, Carlsbad, CA) was used for the synthesis of cNDA. GapA, as a housekeeping gene, was used to determine the levels of mRNA 
transcription of the C. elegans immune genes and to normalize the input amounts of RNA. PCR primers specific to each of the genes were experimentally validated and used in the RT-qPCR assay (Table S9). One $\mu$ l of each cDNA sample was included in a $24 \mu \mathrm{l}$ reaction mixture containing $12.5 \mu \mathrm{l}$ Master Mix, $3.75 \mu \mathrm{l}$ each of the primers at $150 \mathrm{nM}$, and $4 \mu \mathrm{l}$ irradiated and double autoclaved $\mathrm{dH}_{2} \mathrm{O}$. The QPCR programs included $10 \mathrm{~min}$ at $95^{\circ} \mathrm{C}$ and 40 cycles of $95^{\circ} \mathrm{C}$ for $30 \mathrm{~s}, 56^{\circ} \mathrm{C}$ for $1 \mathrm{~min}$, and $72{ }^{\circ} \mathrm{C}$ for $30 \mathrm{~s}$. Fluorescence wasmeasured after each annealing during the cycles. The delta $\mathrm{Ct}$ method was used to analyse the RT-qPCR data and to determine the relative abundance of the target genes (fold changes) (fold changes) $[50,51]$. The data of OP50 group was taken as benchmark 1. All experiment was repeated three times.

\section{Induction of acute iletis for C. jejuni infection and LAB intervention in mice}

Three-week-old female C57BL/6 mice obtained from Shanghai Laboratory Animal Center (Shanghai, China) were used in the experiments. All the experimental procedures (\#IIPD2017030) have been reported [55]. All the experiments conformed to the Ministry of Science and Technology of China's Guide for the Care and Use of Laboratory Animals.

To induce ileitis, the C57BL/6 mice were infected orally with $T$. gondii ME49 cysts according to previous reports $[52,55]$. As shown in Fig. S2A, 4 days later, mice were infected with LAB and C. jejuni NCTC 11168 by gavage at a volume of $0.3 \mathrm{~mL}$ every day individually. The mice were successively treated with LAB $\left(10^{9} \mathrm{CFU}\right)$ and C. jejuni $\left(10^{9} \mathrm{CFU}\right)$ at $1 \mathrm{~h}$ intervals on 2 consecutive days. The $C$. jejuni loads in their faeces were checked on day 9. The faeces were resuspended in sterile PBS and serially diluted. The diluted samples were spread on Columbia blood agar with a $C$. jejuni selective supplement and incubated at $5 \%$ oxygen concentration at $37^{\circ} \mathrm{C}$ for $48 \mathrm{~h}$. After incubation, the numbers of C. jejuni in the samples were counted. The mice were euthanized with $\mathrm{CO}_{2}$ after experiment. For each mice, the treatment based on the different LAB, the blood collection and execution and analysis of indicators were all done by different investigator. The first investigator was the only person aware of the treatment group allocation. All experiment was repeated three times.

\section{C. jejuni infection and LAB intervention in chicken}

White leghorn chicken eggs (Jinan Baizhun Biologic Inspection Company, Ltd., China) were maintained in an egg incubator until the chicks hatched. About $24 \mathrm{~h}$ after hatching, 8 chicks were randomly assigned to several groups. All the experimental procedures were approved by the Animal Care and Use Committee at Jiangsu Nannong Hi-technology company, LTD. All the experiments adhered to the Ministry of Science and Technology of China's Guide for the Care and Use of Laboratory Animals.

As shown in Fig. S2B, all birds were administered $10^{9}$ CFU of C. jejuni NCTC 11168 in a $0.3 \mathrm{~mL}$ suspension by oral gavage. Twenty-four hours after oral gavage, LAB $\left(10^{9} \mathrm{CFU}\right.$ in $\left.0.3 \mathrm{~mL}\right)$ were orally administered daily to 11 groups (in total 88 birds) of C. jejuni-inoculated birds for two weeks. PBS was administered to the remaining one $C$. jejuni group of birds (8 birds). Chicks were euthanized with $\mathrm{CO}_{2}$ at $20 \%$ chamber replacement rate on 23 days post-inoculation, and the cecal contents were diluted in ice-cold PBS to $0.1 \mathrm{~g} / \mathrm{mL}$. Ten-fold serial dilutions of each sample were prepared and then plated on Columbia blood agar with a $C$. jejuni selective supplement and incubated at a $5 \%$ oxygen concentration at $37^{\circ} \mathrm{C}$ for $48 \mathrm{~h}$. For each chicken, the treatment based on the different $\mathrm{LAB}$, the blood collection and execution and analysis of indicators were all done by different investigator. The first investigator was the only person aware of the treatment group allocation. All experiment was repeated three times.

\section{Statistical analysis}

Kaplan-Meier survival analysis was used to assess the survival rate of C. elegans. The significance of differences on data was determined by using Student's t-test. Means \pm standard deviations (SDs) were used as a form of data pression. Differenceswere considered significant at a $P$ value of $<0.01$.

\section{Abbreviations}

C. jejuni: Campylobacter jejuni; LAB: lactic acid bacteria; E. coll: Escherichia coli; C. elegans: Caenorhabditis elegans; T. gondii: Toxoplasma gondii

\section{Supplementary Information}

The online version contains supplementary material available at https://doi. org/10.1186/s12866-021-02226-x.

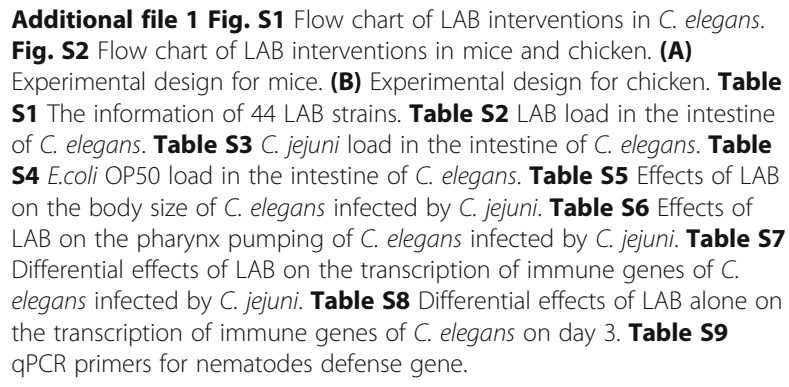

Additional file 1 Fig. S1 Flow chart of $L A B$ interventions in C. elegans. Fig. S2 Flow chart of $L A B$ interventions in mice and chicken. (A) Experimental design for mice. (B) Experimental design for chicken. Table S1 The information of $44 \angle A B$ strains. Table S2 $L A B$ load in the intestine of $C$. elegans. Table S3 $C$. jejuni load in the intestine of $C$. elegans. Table S4 E.coli OP50 load in the intestine of C. elegans. Table S5 Effects of LAB on the body size of $C$. elegans infected by $C$. jejuni. Table S6 Effects of LAB on the pharynx pumping of $C$. elegans infected by $C$. jejuni. Table S7 Differential effects of $L A B$ on the transcription of immune genes of $C$. elegans infected by $C$. jejuni. Table S8 Differential effects of LAB alone on the transcription of immune genes of C. elegans on day 3. Table $\mathbf{S 9}$ qPCR primers for nematodes defense gene.

\section{Acknowledgments}

Not applicable.

Authors' contributions

GW and XJ conceived and designed the experiments; XJ, YH and XC performed the experiments; $X J$ and $G W$ analyzed the data; $Y Z, Y L, J Z, H Z$ and WC contributed reagents/materials/analysis tools; XJ and GW wrote the 
paper. All authors contributed to manuscript revision, read and approved the submitted version.

\section{Funding}

This work was supported by the National Natural Science Foundation of China (No. 31671839, 31972052, 31820103010, 31601444, 31301407), the Fundamental Research Funds for the Central Universities (JUSRP22006, JUSRP51501), the national first-class discipline program of Food Science and Technology (JUFSTR20180102), the Program of Collaborative Innovation Centre of Food Safety and Quality Control in Jiangsu Province and the research supported by Hunan Provincial Natural Science Foundation of China (2019JJ50014) who approved the design of the study and collection, analysis, interpretation of data and final manuscript.

\section{Availability of data and materials}

The datasets used and/or analysed during the current study are available from the corresponding author on reasonable request.

\section{Declarations}

\section{Ethics approval and consent to participate}

All the mice experimental procedures (\#JIPD2017030) had been approved by the Animal Care and Use Committee at the Jiangsu Institute of Parasitic Diseases. All the chicken experimental procedures were approved by the Animal Care and Use Committee at Jiangsu Nannong Hi-technology company, LTD. All the experiments adhered to the Ministry of Science and Technology of China's Guide for the Care and Use of Laboratory Animals.

\section{Consent for publication}

Not applicable.

\section{Competing interests}

The authors declare that they have no competing interests.

\section{Author details}

${ }^{1}$ State Key Laboratory of Food Science and Technology, Jiangnan University, Wuxi 214122, P. R. China. ${ }^{2}$ School of Food Science and Technology, Jiangnan University, Wuxi 214122, P. R. China. ${ }^{3}$ Key Laboratory of National Health and Family Planning Commission on Parasitic Disease Control and Prevention, Jiangsu Provincial Key Laboratory on Parasite and Vector Control Technology, Jiangsu Institute of Parasitic Diseases, Wuxi 214064, P. R. China. ${ }^{4}$ College of Life Sciences and Environment, Hengyang Normal University, Hengyang 421008 , P. R. China. ${ }^{5}$ Department of Microbiology \& Immunology, National University of Singapore, Singapore 117597, Singapore. ${ }^{6}$ International Joint Research Laboratory for Probiotics, Jiangnan University, Wuxi 214122, P. R. China. ${ }^{7}$ Yangzhou) Institute of Food Biotechnology, Jiangnan University, Yangzhou 225004, P. R. China. ${ }^{8}$ National Engineering Research Center for Functional Food, Jiangnan University, Wuxi 214122, P. R. China. ${ }^{9}$ Wuxi Translational Medicine Research Center and Jiangsu Translational Medicine Research Institute Wuxi Branch, Wuxi 214122, P. R. China. ${ }^{10}$ Beijing Innovation Centre of Food Nutrition and Human Health, Beijing Technology and Business University (BTBU), Beijing 100048, P. R. China.

\section{Received: 27 September 2020 Accepted: 13 May 2021} Published online: 05 June 2021

\section{References}

1. Dasti Jl, Tareen AM, Lugert R, Zautner AE, Gross U. Campylobacter jejuni: a brief overview on pathogenicity-associated factors and disease-mediating mechanisms. Int J Med Microbiol. 2010;300(4):205-11. https://doi.org/10.101 6/j.jimm.2009.07.002.

2. Sean F, Altekruse NJS, Patricia I. Fields. Campylobacter jejuni-an emerging foodborne pathogen. Emerg Infect Dis. 1999;5(1):28-35.

3. Zilbauer M, Dorrell N, Wren BW. Campylobacter jejuni-mediated disease pathogenesis: an update. Trans R Soc Trop Med Hyg. 2008;102(2):123-9. https://doi.org/10.1016/j.trstmh.2007.09.019.

4. Alfredson DA, Korolik V. Antibiotic resistance and resistance mechanisms in Campylobacter jejuni and Campylobacter coli. FEMS Microbiol Lett. 2007; 277(2):123-32. https://doi.org/10.1111/j.1574-6968.2007.00935.x.
5. Marie F, Bernet C, Dominique B. The human Lactobacillus acidophilus strain LA1 secretes a Nonbacteriocin antibacterial substance(s) active in vitro and in vivo. Appl Environ Microbiol. 1997;63(7):2747-53.

6. Waard JG, Vos JG. Antagonistic activity of Lactobacillus casei strain Shirota against gastrointestinal Listeria monocytogenes infection in rats. Int J Food Microbiol. 2002;73(2):93-100. https://doi.org/10.1016/S0168-1605(01)00699-7.

7. Nishiyama K, Seto Y, Yoshioka K. Lactobacillus gasseri SBT2055 reduces infection by and colonization of Campylobacter jejuni. PLoS One. 2014;9(9): $1-9$.

8. Cole M, Donoghue AM. Bacteriocins reduce Campylobacter colonization and Alter gut morphology in Turkey Poults. Poult Sci. 2006;85(2):1570-5. https:// doi.org/10.1093/ps/85.9.1570.

9. Wagner RD, Johnson SJ, Kurniasih RD. Probiotic bacteria are antagonistic to Salmonella enterica and Campylobacter jejuni and influence host lymphocyte responses in human microbiota-associated immunodeficient and immunocompetent mice. Mol Nutr Food Res. 2009;53(3):377-88. https://doi. org/10.1002/mnfr.200800101.

10. Man-Wah TS, Frederick M. Killing of Caenorhabditis elegans by Pseudomonas aeruginosa used to model mammalian bacterial pathogenesis. Microbiol. 1999;96(3):715-20.

11. Sifri CD, Begun J, Ausubel FM. Caenorhabditis elegans as a model host for Staphylococcus aureus pathogenesis. Infect Immun. 2003;71(4):2208-17. https://doi.org/10.1128/IAl.71.4.2208-2217.2003.

12. Alejandro AP. Salmonella typhimurium proliferates and establishes a persistent infection in the intestine of Caenorhabditis elegans. Curr Biol. 2000;10(23):1539-42.

13. Ikeda T, Yasui C, Hoshino K. Influence of lactic acid bacteria on longevity of Caenorhabditis elegans and host defense against salmonella enterica serovar enteritidis. Appl Environ Microbiol. 2007;73(20):6404-9. https://doi.org/10.112 8/AEM.00704-07.

14. Garsin DA, Sifri CD, Mylonakis E. A simple model host for identifying grampositive virulence factors. Proc Natl Acad Sci. 2001;98(19):10892-7. https:// doi.org/10.1073/pnas.191378698.

15. Terence I, Moy ALC, Jonah L. High-throughput screen for novel antimicrobials using a whole animal infection model. ACS Chem Biol. 2009; 4(7):527-33.

16. Cean $A$, Stef $L$, Simiz E. Effect of human isolated probiotic bacteria on preventing Campylobacter jejuni colonization of poultry [J]. Foodborne Pathog Dis. 2015;12(2):122-30. https://doi.org/10.1089/fpd.2014.1849.

17. Durai S, Pandian SK, Balamurugan K. Establishment of a Caenorhabditis elegans infection model for Vibrio alginolyticus. J Basic Microbiol. 2011;51(3): 243-52. https://doi.org/10.1002/jobm.201000303.

18. Kamaladevi A, Balamurugan K. Role of PMK-1/p38 MAPK defense in Caenorhabditis elegans against Klebsiella pneumoniae infection during hostpathogen interaction. Pathog Dis. 2015;73(5):1-9.

19. JebaMercy G, Vigneshwari L, Balamurugan K. A MAP kinase pathway in Caenorhabditis elegans is required for defense against infection by opportunistic Proteus species. Microbes Infect. 2013;15(8-9):550-68. https:// doi.org/10.1016/j.micinf.2013.03.009.

20. Grompone G, Martorell P, Llopis S. Anti-inflammatory Lactobacillus rhamnosus CNCM I-3690 strain protects against oxidative stress and increases lifespan in Caenorhabditis elegans. PLoS One. 2012;7(12):1-13.

21. Kim Y, Mylonakis E. Caenorhabditis elegans immune conditioning with the probiotic bacterium Lactobacillus acidophilus strain NCFM enhances grampositive immune responses. Infect Immun. 2012;80(7):2500-8. https://doi. org/10.1128/IAl.06350-11.

22. Jin LH, Kyu WC. Evaluation of probiotic characteristics of newly isolated Lactobacillus spp immune modulation and longevity. Int J Food Microbiol. 2011:148(2):80-6.

23. Bolton DJ. Campylobacter virulence and survival factors. Food Microbiol. 2015;48:99-108. https://doi.org/10.1016/j.fm.2014.11.017.

24. Wieczorek K, Osek J. A five-year study on prevalence and antimicrobial resistance of Campylobacter from poultry carcasses in Poland. Food Microbiol. 2015;49:161-5. https://doi.org/10.1016/j.fm.2015.02.006.

25. Baffoni L, Gaggia F, Di Gioia D, Santini C, Mogna L, Biavati B. A Bifidobacterium-based synbiotic product to reduce the transmission of $C$. jejuni along the poultry food chain. Int J Food Microbiol. 2012;157(2):156-61. https://doi.org/10.1016/j.jffoodmicro.2012.04.024.

26. Messaoudi S, Manai M, Kergourlay G, Prevost H, Connil N, Chobert JM, et al. Lactobacillus salivarius: bacteriocin and probiotic activity. Food Microbiol. 2013;36(2):296-304. https://doi.org/10.1016/j.fm.2013.05.010. 
27. Zhou M, Yu H, Yin X. Lactobacillus zeae protects Caenorhabditis elegans from enterotoxigenic Escherichia coli-caused death by inhibiting enterotoxin gene expression of the pathogen. PLoS One. 2014;9(2):1-10.

28. Barbara Caleen Hansen NLB, Heidi K, Ortmeyer. Calorie restriction in nonhuman primates: mechanisms of reduced morbidity and mortality. Toxicol Sci. 1999;52(3):56-60. https://doi.org/10.1093/toxsci/52.suppl_1.56.

29. Hine C, Mitchell JR. Calorie restriction and methionine restriction in control of endogenous hydrogen sulfide production by the transsulfuration pathway. Exp Gerontol. 2015;68:26-32. https://doi.org/10.1016/j.exger.2 014.12 .010$.

30. Vera E, Bernardes de Jesus B, Foronda M, Flores JM, Blasco MA. Telomerase reverse transcriptase synergizes with calorie restriction to increase health span and extend mouse longevity. PLoS One. 2013;8(1):e53760.

31. Irazoqui JE, Urbach JM, Ausubel FM. Evolution of host innate defence: insights from Caenorhabditis elegans and primitive invertebrates. Nat Rev Immunol. 2010;10(1):47-58. https://doi.org/10.1038/nri2689.

32. Zhang L, Xu YQ, Liu HY. Evaluation of Lactobacillus rhamnosus GG using an Escherichia coli K88 model of piglet diarrhoea: effects on diarrhoea incidence, faecal microflora and immune responses. Vet Microbiol. 2010; 141(1-2):142-8. https://doi.org/10.1016/j.vetmic.2009.09.003.

33. Miettinen $M$, Veckman V, Latvala S. Live Lactobacillus rhamnosus and streptococcus pyogenes differentially regulate toll-like receptor (TLR) gene expression in human primary macrophages. J Leukoc Biol. 2008;84(4):1092100. https://doi.org/10.1189/jlb.1206737.

34. Vizoso PMG, Rodriguez GM, Seifert S. Lactobacilli stimulate the innate immune response and modulate the TLR expression of HT29 intestinal epithelial cells in vitro. Int J Food Microbiol. 2009;133(1-2):86-93. https://doi. org/10.1016/j.ijfoodmicro.2009.05.013.

35. Wachi S, Kanmani P, Tomosada Y. Lactobacillus delbrueckii TUA4408L and its extracellular polysaccharides attenuate enterotoxigenic Escherichia coliinduced inflammatory response in porcine intestinal epitheliocytes via tolllike receptor-2 and 4. Mol Nutr Food Res. 2014;58(10):2080-93. https://doi. org/10.1002/mnfr.201400218.

36. Fang SB, Shih HY, Huang CH, Li LT, Chen CC, Fang HW. Live and heat-killed Lactobacillus rhamnosus GG upregulate gene expression of proinflammatory cytokines in 5-fluorouracil-pretreated Caco-2 cells. Support Care Cancer. 2014;22(6):1647-54. https://doi.org/10.1007/s00520-014-2137-z.

37. Nandakumar NS, Pugazhendhi S, Madhu Mohan K, Jayakanthan K, Ramakrishna BS. Effect of Vibrio cholerae on chemokine gene expression in HT29 cells and its modulation by Lactobacillus GG. Scand J Immunol. 2009; 69(3):181-7. https://doi.org/10.1111/j.1365-3083.2008.02214.x.

38. Mallo GV, CLo K. Carole Couillault inducible antibacterial defense system in C. elegans. Curr Biol. 2003;12(3):1209-14.

39. Wang G, Xu Q, Jin X, Hang F, Liu ZM, Zhao JX, et al. Effects of lactobacilli with different regulatory behaviours on tight junctions in mice with dextran sodium sulphate-induced colitis. J Funct Foods. 2018;47:107-15. https://doi. org/10.1016/j.jf.2018.05.044.

40. Pujol N, Zugasti O, Wong D. Anti-fungal innate immunity in C. elegans is enhanced by evolutionary diversification of antimicrobial peptides. PLoS Pathog. 2008;4(7):1-12.

41. Mertenskotter A, Keshet A, Gerke P, Paul RJ. The p38 MAPK PMK-1 shows heat-induced nuclear translocation, supports chaperone expression, and affects the heat tolerance of Caenorhabditis elegans. Cell Stress Chaperones. 2013;18(3):293-306. https://doi.org/10.1007/s12192-012-0382-y.

42. Roh JY, Eom HJ, Choi J. Involvement of Caenohabditis elegans MAPK signaling pathways in oxidative stress response induced by silver nanoparticles exposure. Toxicol Res. 2012;28(1):19-24. https://doi.org/10.54 87/TR.2012.28.1.019.

43. Uno M, Honjoh S, Matsuda M, Hoshikawa H, Kishimoto S, Yamamoto T, et al. A fasting-responsive signaling pathway that extends life span in C. elegans. Cell Rep. 2013;3(1):79-91. https://doi.org/10.1016/j.celrep.2012.12.01 8.

44. Vigneshkumar B, Pandian SK, Balamurugan K. Regulation of Caenorhabditis elegans and Pseudomonas aeruginosa machinery during interactions. Arch Microbiol. 2012;194(4):229-42. https://doi.org/10.1007/s00203-011-0750-5.

45. Ausubel FM, Hoeven VD. Ransome. Ce-Duox1/BLI-3 generated reactive oxygen species trigger protective SKN-1 activity via p38 MAPK signaling during infection in C. elegans. PLoS Pathog. 2011;7(12):1-11.

46. Hoeven R, McCallum KC, Cruz MR. Ce-Duox1/BLI-3 generated reactive oxygen species trigger protective SKN-1 activity via p38 MAPK signaling during infection in C. elegans. PLoS Pathog. 2011;7(12):1-14.
47. Ristow M, Senchuk MM, Dues DJ, Schaar CE, Johnson BK, Madaj ZB, et al. Activation of DAF-16/FOXO by reactive oxygen species contributes to longevity in long-lived mitochondrial mutants in Caenorhabditis elegans. PLoS Genet. 2018;14(3):1-27.

48. Stiernagle T. Maintenance of C. elegans. In: WormBook; 2006. p. 1-11.

49. Yin X, Chambers JR, Wheatcroft R. Adherence of Escherichia coli O157:H7 mutants in vitro and in ligated pig intestines. Appl Environ Microbiol. 2009; 75(15):4975-83. https://doi.org/10.1128/AEM.00297-09.

50. Zhu J, Yin $\mathrm{X}, \mathrm{Yu} \mathrm{H}$. Involvement of quorum sensing and heat-stable enterotoxin a in cell damage caused by a porcine enterotoxigenic Escherichia coli strain. Infect Immun. 2011;79(4):1688-95. https://doi.org/1 0.1128/IAl.01281-10.

51. Zhou M, Zhu J, Yu H, Yin X, Sabour PM, Zhao L, et al. Investigation into in vitro and in vivo models using intestinal epithelial IPEC-J2 cells and Caenorhabditis elegans for selecting probiotic candidates to control porcine enterotoxigenic Escherichia coli. J Appl Microbiol. 2014;117(1):217-26. https://doi.org/10.1111/jam.12505.

52. Haag LM, Fischer A, Otto B. Intestinal microbiota shifts towards elevated commensal Escherichia coli loads abrogate colonization resistance against Campylobacter jejuni in mice. PLoS One. 2012;7(5):1-13.

53. Wang G, Zhao Y, Tian FW, Jin X, Chen HQ, Liu XM, et al. Screening ofadhesive lactobacilli with antagonistic activity against campylobacter jejuni. Food Control. 2014;44(44):49-57. https://doi.org/10.1016/j.foodcont.2 014.03.042.

54. Piper C, Casey PG, Hill C. The lantibiotic lacticin 3147 prevents systemic spread of Staphylococcus aureusin a murine infection model [J]. Int J Microbiol. 2012;11(1):129-37.

55. Jin X, He YF, Liu ZM, Zhou YH, Chen XH, Wang G, et al. Lactic acid bacteria exhibit similar antioxidant capacities in Caenorhabditis elegans- and Campylobacter jejuni-infected mice [J]. RSC Adv. 2020;10(6):3329-42. https:// doi.org/10.1039/C9RA06105C

\section{Publisher's Note}

Springer Nature remains neutral with regard to jurisdictional claims in published maps and institutional affiliations.

\section{Ready to submit your research? Choose BMC and benefit from:}

- fast, convenient online submission

- thorough peer review by experienced researchers in your field

- rapid publication on acceptance

- support for research data, including large and complex data types

- gold Open Access which fosters wider collaboration and increased citations

- maximum visibility for your research: over $100 \mathrm{M}$ website views per year

At BMC, research is always in progress.

Learn more biomedcentral.com/submissions 